\title{
miR-512-5p induces apoptosis and inhibits glycolysis by targeting p21 in non-small cell lung cancer cells
}

\author{
KAILI CHU ${ }^{1 *}$, GUANGHUI GAO ${ }^{2 *}$, XIUFANG YANG ${ }^{1}$, SHENGXIANG REN $^{2}$, \\ YAO $\mathrm{LI}^{1}$, HAI WU ${ }^{1}$, YAN HUANG ${ }^{1}$ and CAICUN ZHOU ${ }^{2}$ \\ ${ }^{1}$ State Key Laboratory of Genetic Engineering, Institute of Genetics, School of Life Sciences, Fudan University, \\ Shanghai 200438; ${ }^{2}$ Department of Oncology, Shanghai Pulmonary Hospital, Tongji University \\ School of Medicine, Tongji University Cancer Institute, Shanghai 200433, P.R. China
}

Received October 11, 2015; Accepted November 12, 2015

DOI: $10.3892 /$ ijo.2015.3279

\begin{abstract}
MicroRNAs are a family of small non-coding RNAs that constitute a prevalent gene regulation. In this study, we showed the expression of miR-512-5p is downregulated in non-small cell lung cancer (NSCLC) patient tumor samples compared to its paired normal lung tissues. Moreover, expression of miR-512-5p was increased by retinoic acid treatment. Overexpression of miR-512-5p induced apoptosis of NSCLC cell lines A549 and H1299, and miR-512-5p inhibitor reversed this effect in H1299 cells stably expressing miR-512. miR$512-5 p$ inhibited glycolysis and migration in NSCLC cells, but shows no effect on cell proliferation. We identified p21 as a target gene of miR-512-5p. Overexpression of miR-512-5p led to the decrease of p21 protein and mRNA level. Knockdown of p21 resulted in similar effects on apoptosis and glycolysis as that observed of miR-512-5p overexpression, as well as rescued the effect of miR-512-5p inhibitor on cell apoptosis in H1299 cells stably expressing miR-512. In conclusion, our present study revealed miR-512-5p was able to target $\mathrm{p} 21$ to induce apoptosis and inhibit glycolysis in A549 and H1299 cell lines.
\end{abstract}

\section{Introduction}

Lung cancer is the most frequently diagnosed cancer and remains the leading cause of cancer death among males, and has surpassed breast cancer as the leading cause of cancer death among females in most developed countries (1). Currently, the typical non-small cell lung cancer (NSCLC) patients account for $85 \%$ of the total lung cancer patients (2).

Correspondence to: Professor Yan Huang, State Key Laboratory of Genetic Engineering, Institute of Genetics, School of Life Sciences, Fudan University, Shanghai 200438, P.R. China

E-mail: huangyan@fudan.edu.cn

*Contributed equally

Key words: miR-512-5p, p21, apoptosis, glycolysis, migration
miRNAs are a family of evolutionarily conserved small non-coding RNAs that constitute a prevalent gene regulation, they often block mRNA translation or negatively regulate its stability through binding site within the 3'-UTR of target mRNA (3). Recent studies suggested that miRNA appears to be a key determinant in diagnosis and prognosis of many diseases. miRNAs associated with tumorigenesis display either tumor-promoting properties or tumor-suppressing characteristics. For example, miR-212 in lung cancer (4) and the miR-17-92 cluster in malignant lymphoma (5) act as oncogenes. In contrast, let-7 in lung cancer (6) and miR-551a/ miR-483 in colorectal cancer (7) act as tumor suppressors.

Retinoic acid (RA), the major bioactive metabolite of retinol or vitamin A, plays critical roles in cell growth, differentiation and cell apoptosis (8). It has been successfully employed for decades in the treatment of acute promyelocytic leukemia (9). In recent years, it was reported that RA could regulate the expression level of miRNAs in cancer, such as in breast cancer cells $(10,11)$. miR-512-5p is located at chromosome $19 \mathrm{q} 13.42$ and is a member of miR-512 cluster, which includes two copies of miR-512 (miR-512-1 and miR-512-2) and 46 duplicates of miR-519 (12). From previous observations and studies, miR-512-5p is significantly upregulated in cervical intraepithelial neoplasia specimens compared to normal cervical epithelium samples (13). Activation of miR$512-5 p$ by epigenetic treatment induces apoptosis of gastric cancer cells by suppressing Mcl-1 (14).

$\mathrm{p} 21^{\mathrm{WAF} / \mathrm{CIP} 1}$ is located at chromosome $6 \mathrm{p} 21.2$, and it belongs to the Cip /Kip family of CKIs (15). Early studies supported the view that p21 is a master effector of the p53-dependent G1 arrest (16). p21 regulates its various biological activities primarily by binding to and inhibiting the cyclin/CDK activity, leading to growth arrest in the cell cycle (17). However, recent studies also showed that $\mathrm{p} 21$ has context-dependent function in apoptosis (18).

Based on the present investigation we report that miR$512-5 \mathrm{p}$ is downregulated in lung cancer tissues versus paired normal lung tissues. Overexpression of miR-512-5p induced cell apoptosis, and inhibited glycolysis in NSCLC cells. Further evidence showed that p21 was the target of miR$512-5 p$, whose downregulation may be responsible for the function of miR-512-5p. 


\section{Materials and methods}

Cell culture. A549, H1299 (American Type Culture Collection, Manassas, VA, USA) and bulk-selected H1299 were cultured in RPMI-1640 medium (Gibco, Grand Island, NY, USA), supplemented with 10\% FBS (Biowest, Barcelona, Catalonia, Spain), $1 \%$ penicillin and streptomycin and $1 \%$ sodium pyruvate (Gibco) at $37^{\circ} \mathrm{C}$ in a humidified atmosphere of $5 \% \mathrm{CO}_{2}$.

Human tissue samples. Twelve NSCLC tumor samples and their corresponding normal tissues were collected from Shanghai Pulmonary Hospital, snap-frozen in liquid nitrogen and stored at $-70^{\circ} \mathrm{C}$ until analysis, between August 2012 and January 2014. None of the patients received pre-surgical chemo/radiation therapy. All NSCLC patients gave written informed consent for the use of clinical specimens for medical research. The study was reviewed and approved by the Committee for Ethical Review of Research Involving Human Subjects at Shanghai Pulmonary Hospital.

Expressing constructs. The pEGP-miR-512 construct was cloned as described (19). In brief, the genomic fragment of hsa-miR-512 precursor was amplified. The PCR primers were: 5'-TCGAGGATCCACCTTTCACTATTGAGCA ACA-3' and 5'-TCGAGCTAGCTGAAGTCCAGTTTAT GGCGCA-3'. The PCR product was digested with EcoRI and NheI restriction enzymes and cloned into the pEGP-miR vector (Cell Biolabs, San Diego, CA, USA). The construct was designated as pEGP-miR-512.

Transient transfection and bulk-selected $H 1299$ cells. All transfection were carried out using Lipofectamine ${ }^{\mathrm{TM}} 2000$ (Invitrogen, Carlsbad, CA, USA) according to the manufacturer's procedure. For H1299 cells stably expressing miR-512, H1299 cells transfected with pEGP-miR-512 were selected with $5 \mu \mathrm{g} / \mathrm{ml}$ puromycin (Sigma, St. Louis, MO, USA) for two weeks. The bulk-selected H1299 cells stably expressing miR-512 were maintained in RPMI-1640 (Gibco) with $2.5 \mu \mathrm{g} / \mathrm{ml}$ puromycin (Gibco). Bulk cultures were used to avoid clone specific effects.

RNA isolation and quantitative RT-PCR. Total RNA was extracted with Trizol (Invitrogen), and miRNA was extracted with miRcute miRNA Isolation kit (Tiangen, Shanghai, China). The RNA was converted to cDNA using Primer Script RT Reagent kit (Takara, Tokyo, Japan), which was incubated for $15 \mathrm{~min}$ at $37^{\circ} \mathrm{C}$ followed by $5 \mathrm{~min}$ at $85^{\circ} \mathrm{C}$. The specific reverse transcription (RT) primer of miR-512-5p was 5'-GTCGTATCCAGTGCAGGGTCCGAGGTATTCGC ACTGGATACGACGAAAGTG-3'. Real-time quantitative PCR was run on a Light-Cycler Roche 480 (Roche Applied Science, Shanghai, China) with reactions consisted of a hot $\operatorname{start}\left(10 \mathrm{~min}\right.$ at $\left.95^{\circ} \mathrm{C}\right)$, followed with 40 cycles of $15 \mathrm{sec}$ at $95^{\circ} \mathrm{C}$ and $60 \mathrm{sec}$ at $60^{\circ} \mathrm{C}$. The expression level of miR-512-5p and p21 was normalized to U6 and GAPDH, respectively. Each sample was run in triplicate to ensure accuracy. The PCR primers for miR-512-5p were 5'-CGGCGGCACTCAGCCTTGAGGG-3' and 5'-GTGCAGGGTCCGAGGT-3'. The PCR primers for p21 were 5'-CTACTGAGGAGCCAGCGTCTA-3' and

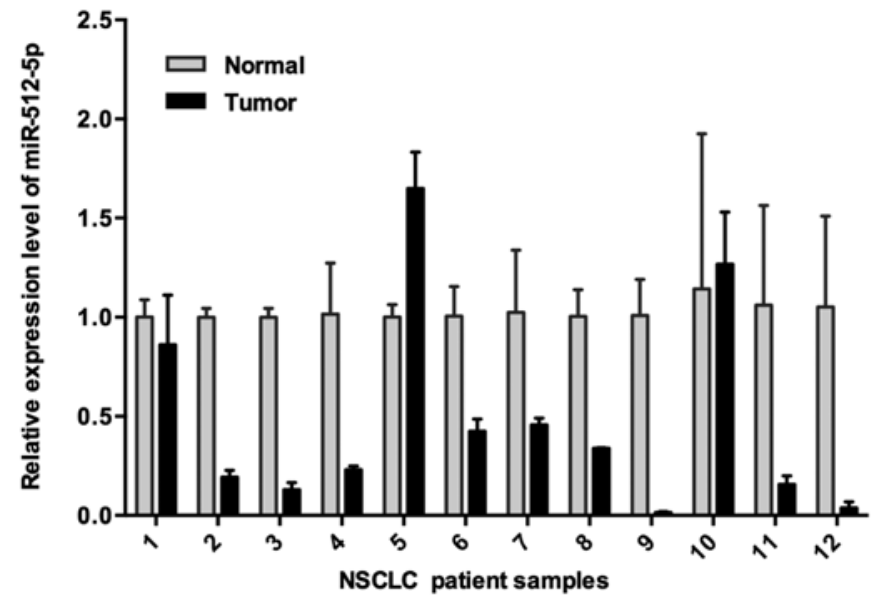

Figure 1. Expression of miR-512-5p was downregulated in NSCLC patient samples. Relative miR-512-5p expression was detected by real-time PCR in 12 paired normal and tumor tissues. Relative miR-512-5p expression levels in NSCLC tumors were presented as-fold change of tumor versus paired normal tissues, and the expression level of normal tissues was assigned as 1.0. Expression of miR-512-5p was downregulated in most of the tissue samples $(10 / 12)$. Each experiment was repeated three times. Data were normalized to $\mathrm{U} 6$ and error bars represented mean $\pm \mathrm{SD}$.

5'-CTGCCCATCATCATGACCT-3'. The PCR primers for GAPDH were 5'-CTTAGATTTGGTCGTATTGG-3' and 5'-GAAGATGGTGATGGGATT-3'.

The miRNA mimics, miRNA inhibitor and siRNA. miR-512-5p mimic and miR-512-5p inhibitor were synthesized by Biotend (Shanghai, China), and p21 siRNA were synthesized by GenePharma (Shanghai, China). The sequence of miR-512-5p inhibitor was 5'-GAAAGUGCCCUCAAGGCUGAGUG-3'. Two pairs of p21 siRNA were synthesized. The sequences of p21 siRNA-1 (sip21-1) were 5'-AAUGGCGGGCUGCAU CCAGGA-3' and 5'-UCCUGGAUGCAGCCCGCCAUU-3', and p21 siRNA-2 (sip21-2) were 5'-CUUCGACUUUGUCA CCGAG-3' and 5'-CUCGGUGACAAAGUCGAAG-3'.

Cell apoptosis assay. Apoptosis rate was assessed by the FITC-Annexin V/PI Apoptosis Detection kit (BD Pharmingen, San Diego, CA, USA) according to the manufacturer's instruction. At $48 \mathrm{~h}$ after transfection, cells were collected and assayed with Annexin V binding buffer containing FITC-Annexin V and PI for $15 \mathrm{~min}$ at $37^{\circ} \mathrm{C}$ in the dark. Cells were analyzed by a flow cytometer (BD FACSCalibur, BD Bioscience, San Jose, CA, US) and CellQuest software.

Glucose consumption and lactate production. Cells were seeded on a 12-well plate well, and the culture medium was changed to RPMI-1640 medium without FBS $4 \mathrm{~h}$ after transfection. The concentrations of glucose and L-lactate were measured after another $36 \mathrm{~h}$ of incubation with a glucose test kit (Sigma) and L-lactate assay kit (Sigma), respectively.

Cell proliferation assay. Cell Counting Kit-8 (Dojindo Laboratories, Kumamoto, Japan) was used to perform cell proliferation assays according to the protocol of the manufacturers on days 1, 2, 3 and 4 post-plating. Briefly, 5000 cells were plated in 96-well culture plate per well and were incubated 
A

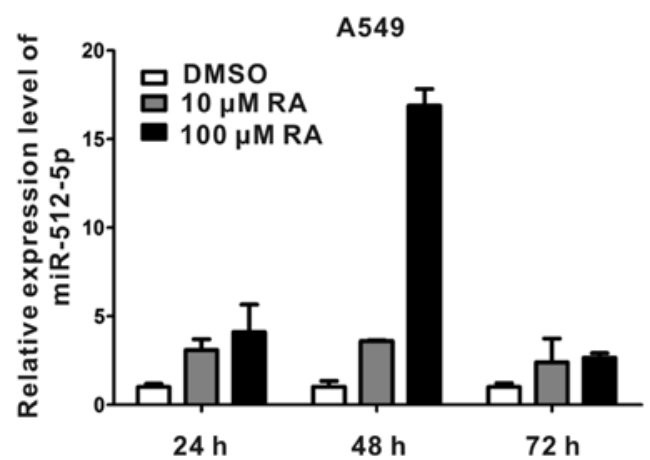

B

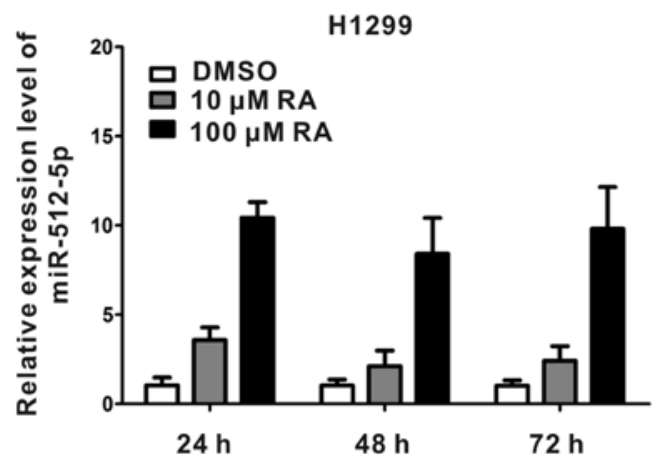

Figure 2. Expression of miR-512-5p in A549 and H1299 cells by Retinoic Acid (RA) treatment. (A) Real-time PCR showed that in A549 cells, expression of miR-512-5p increased $~ 3$-fold compared with that of control (DMSO) when exposed to $10 \mu \mathrm{M}$ RA. As the concentration of RA expanded to $100 \mu \mathrm{M}$, the expression of miR-512-5p increased 5, 17 and 3-fold at 24 h, 48 and 72 h, respectively. (B) In H1299 cells, the expression of miR-512-5p increased less than 4-fold when under $10 \mu \mathrm{M}$ RA and increased to about 10-fold when exposed to $100 \mu \mathrm{M}$ RA. Each experiment was performed in triplicate and error bars represent mean $\pm \mathrm{SD}$.

in $10 \mu \mathrm{l} \mathrm{CCK}-8$ per well for $2 \mathrm{~h}$, then examined. Absorbance was measured at 450 and $630 \mathrm{~nm}$ (used as reference) with a Microplate Reader ELx808 (Bio-Tek Instruments, Winooski, VT, USA). Each experiment was performed in sextuplicate.

Cell migration. For cell migration assay, $5 \times 10^{4}$ cells in $0.1 \mathrm{ml}$ media containing $1 \%$ FBS were seeded into the upper chamber, with $0.6 \mathrm{ml}$ of medium (10\% FBS) under the upper chamber. The cells were then cultured for $72 \mathrm{~h}$, and the noninvading cells were wiped off with a cotton swab. Cells under the chamber were fixed with methanol, stained with DAPI (Sigma), and washed with phosphate-buffered saline (PBS). All of the cells on the bottom of the chamber were counted under the microscope.

Dual luciferase assay. H1299 cells were co-transfected with 50 pmol miR-512-5p, $1 \mu \mathrm{g}$ firefly luciferase reporter containing the wild- or mutant-type putative target site in 3' UTR of p21 gene and $0.15 \mu \mathrm{g}$ Renilla luciferase control vector pRL-TK (Promega, Madison, WI, USA). Assays were assessed $48 \mathrm{~h}$ later using a SpectraMax M5 plate reader (Molecular Devices, Sunnyvale, CA, USA). Co-transfections of luciferase report construct and pRL-TK with miR-512-5p or NC were performed with the same procedure.

Western blotting. Immunoblot was performed as follows. Whole cells were lysed in RIPA buffer (CW, Shanghai, China) supplemented with protease inhibitors (100 $\mu \mathrm{g} / \mathrm{ml}$ PMSF, Sigma-Aldrich; Complete Protease Inhibitor Cocktail, Roche) for $15 \mathrm{~min}$. Protein extracted from lysates were submitted to SDS-PAGE (10\%) and transferred to polyvinylidenedifluoride plus membrane (Merck Millipore, Darmstadt, Germany). Membranes were incubated in 5\% non-fat milk prepared with Tris-buffered saline containing $0.1 \%$ Tween-20 (TBST) at room temperature (RT) for $1.5 \mathrm{~h}$, and the membranes were immunoblotted with rabbit anti-p21 polyclonal antibody (1:1000, Proteintech, Chicago, IL, USA) or with mouse antitubulin monoclonal antibody (1:5000, Sigma) overnight at $4^{\circ} \mathrm{C}$, washed two times using TBST at RT, and then incubated in secondary antibodies against rabbit or mouse $\operatorname{IgG}(1: 5000$, KPL, Gaithersburg, MD, USA) for $1.5 \mathrm{~h}$ at RT. The membranes were washed three times and antigen-antibody complexes were visualized by enhanced chemiluminescence (GE Healthcare, Little Chalfont, Buckinghamshire, UK) following the manufacturer's instructions.

Statistical analysis. Data are presented as mean \pm SD of at least three independent experiments for each cellular experimental group. Samples were analyzed by two-tailed unpaired Student'st test, and a $\mathrm{P}<0.05$ was considered statistically significant. We used one, two, and three asterisks in the figures to represent $\mathrm{P}<0.05, \mathrm{P}<0.01$, and $\mathrm{P}<0.001$, respectively. All histograms were drawn using GraphPad Prism 5.

\section{Results}

miR-512-5p is suppressed in human NSCLC tissues. miR$512-5 \mathrm{p}$ is reported to be significantly upregulated in cervical intraepithelial neoplasia specimens compared to normal cervical epithelium samples (13). To assess the expression levels of miR-512-5p in NSCLC, we collected 12 pairs of flash-frozen tumor vs. normal lung tissues from 12 NSCLC patients. Of the 12 NSCLC patients, 9 patients were subtyped as adenocarcinoma, 3 were subtyped as squamous carcinoma (data not shown). Expression of miR-512-5p was examined by RT-qPCR. Relative miR-512-5p expression levels in NSCLC tumors were presented as-fold change $\left(2^{\Delta \mathrm{Ct} \text { tumor- } \Delta \mathrm{Ct} \text { normal }}\right)$ of tumor versus paired normal tissues, expression level of normal tissues was assigned as 1.0. Compared to paired normal tissues, miR-512-5p was repressed in tumor samples in $83 \%(10 / 12)$ of NSCLC cases; while two paired samples showed increased expression of miR-512-5p in tumor tissue (Fig. 1). This result suggested miR-512-5p might act as a tumor suppressor in NSCLC.

$R A$ induces the expression of $m i R-512-5 p$. To explore whether miR-512-5p played a role in the progress of NSCLC cells, we set out to analyze the expression level of miR-512-5p in A549 and H1299 with and without RA treatment at $10 \mu \mathrm{M}$ or $100 \mu \mathrm{M}$ at different time points. Cells treated with dimethyl sulfoxide (DMSO) were used as control. As shown in Fig. 2A, the expression of miR-512-5p increased about 3-fold compared 
A
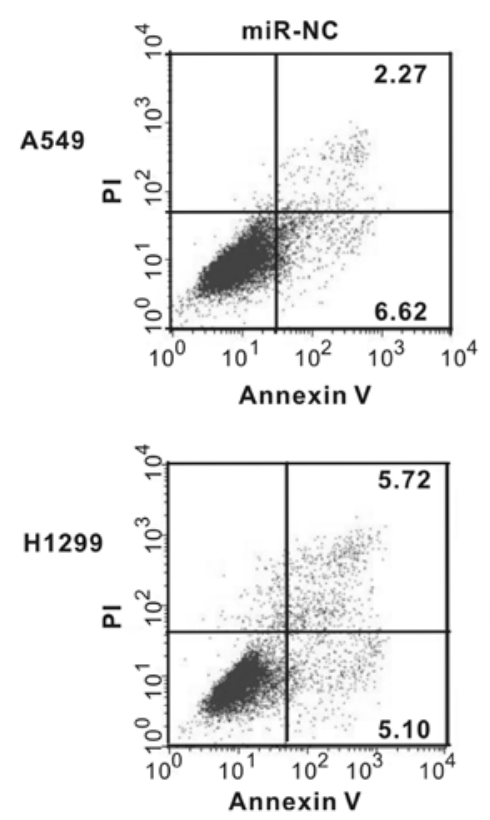
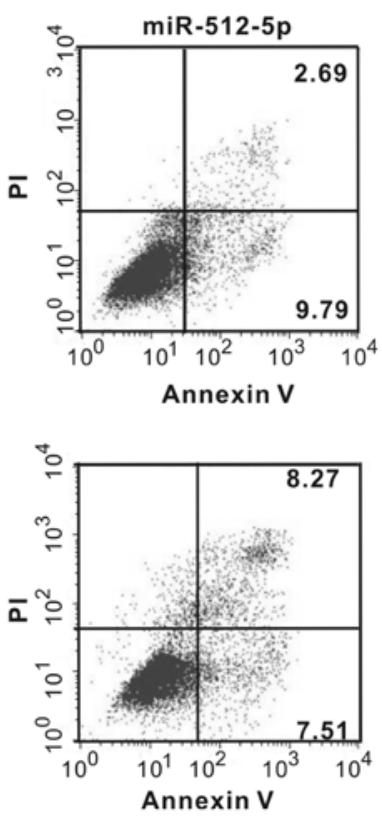

B
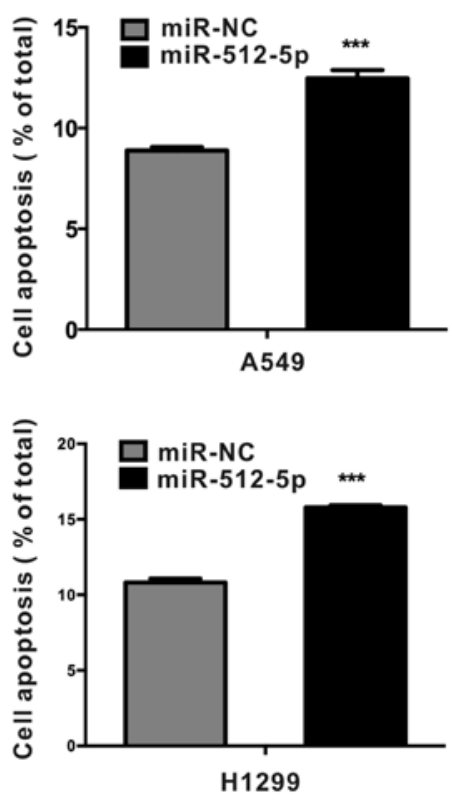

Figure 3. miR-512-5p induces NSCLC cells apoptosis. Apoptosis assays were performed $48 \mathrm{~h}$ after transfection of $50 \mathrm{nM}$ miR-NC or miR-512-5p mimics in A549 cells or H1299 cells. (A) Representative percentage of apoptotic cells using flow cytometric analysis. (B) The histogram shows the apoptotic cell percentage detected by FCM, and error bars denote mean \pm SD. ${ }^{* * *} \mathrm{P}<0.001$.

with that of control when exposed to $10 \mu \mathrm{M}$ RA for 24,48 and $72 \mathrm{~h}$ in A549 cells. As the RA concentration was expanded to $100 \mu \mathrm{M}$, the expression of $\mathrm{miR}-512-5 \mathrm{p}$ increased by $5-$, 17- and 3-fold at 24, 48 and 72 h, respectively. In H1299 cells, the expression of miR-512-5p increased less than 4-fold compared with that of control when treated with $10 \mu \mathrm{M}$ RA. However, when exposed to $100 \mu \mathrm{M}$ RA, the expression level of miR-512-5p increased to about 10-fold at these three time points (Fig. 2B).

Overexpression of miR-512-5p promotes cell apoptosis. A549 and $\mathrm{H} 1299$ cells were cultured for $48 \mathrm{~h}$ after transfection with $50 \mathrm{nM}$ miR-NC or miR-512-5p mimics, then stained with FITC-Annexin V and PI, and analyzed by flow cytometry. We found that cells overexpressing miR-512-5p had a larger population of apoptotic cells than control cells. In A549 cells, overexpression of miR-512-5p caused $40 \%$ increase of apoptotic cells compared with that of control cells. In H1299 cells, the increase of apoptotic cells was about $46 \%$ (Fig. 3).

miR-512-5pinhibits glycolysis in NSCLC cells. The Warburg effect of energy metabolism is critical to the survival and proliferation of cancer cells (20). We asked whether miR512-5p affected cancer cell energy metabolism. To this end, we transfected A549 and H1299 with miR-512-5p and $4 \mathrm{~h}$ later the culture medium was changed to RPMI-1640 without FBS. After another $36 \mathrm{~h}$ of culture, the glucose consumption and lactate production were detected. As shown in Fig. 4, the rates of glucose consumption and lactate production were strongly decreased by miR-512-5p overexpression in A549 and H1299 cells.

miR-512-5p has no effect on cell proliferation. We assessed cell growth rate at day 1, 2, 3 and 4 by CCK- 8 assay to study the role of miR-512-5p on cell proliferation in A549, H1299 and H226 cells. The cells were transfected with either miR-512-5p or miR-NC. Compared with miR-NC, miR-512-5p mimics exhibited almost no effect on cell proliferation on A549, H1299 and H226 cells (Fig. 5).

miR-512-5p attenuates the capacity of cell migration in A549 cells. Metastasis is a process that involves multiple steps, including cancer cell adhesion, invasion and migration (21). To explore the role of miR-512-5p in these processes, we transfected A549 cells with miR-NC or miR-512-5p and seeded cells into the transwell chambers. After incubation for $72 \mathrm{~h}$, medium in chamber was removed and all of the cells on the bottom of the chamber were counted under the microscope. We found overexpression of miR-512-5p attenuated A549 cells migration by $39 \%$ compared to that of control (Fig. 6).

Overexpression of miR-512-5p decreases $p 21 \mathrm{mRNA}$ and protein. In the screening of genes that could be affected by miR-512-5p overexpression, we found that p21 was downregulated by miR-512-5p. A549 and H1299 cells were transiently transfected with miR-NC or miR-512-5p mimics for $48 \mathrm{~h}$, and the expression of $\mathrm{p} 21 \mathrm{mRNA}$ and protein were detected by real-time PCR and western blotting. As shown in Fig. 7A, overexpression of miR-512-5p decreased p21 mRNA by $71 \%$ and $91 \%$ in A549 and H1299 cells, respectively. Compared with cells transfected with miR-NC, p21 protein was decreased significantly in cells transfected with miR-512-5p (Fig. 7B). The experiments showed miR-512-5p inhibited the expression of p21 in NSCLC cells.

p21 is a direct target gene of miR-512-5p. To explore the mechanism of miR-512-5p in downregulating $\mathrm{p} 21$, we first performed 
A

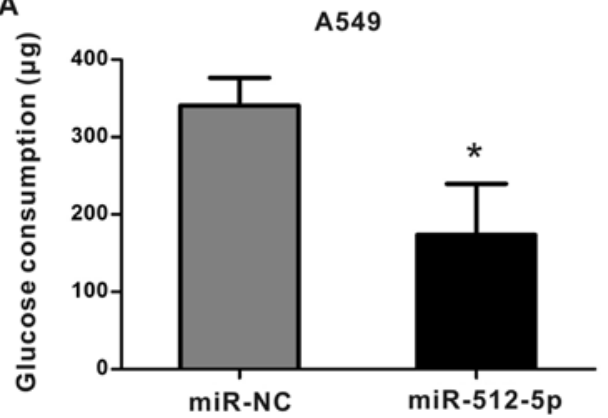

C

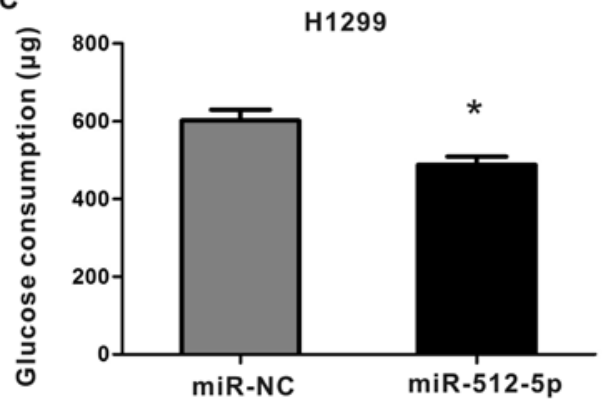

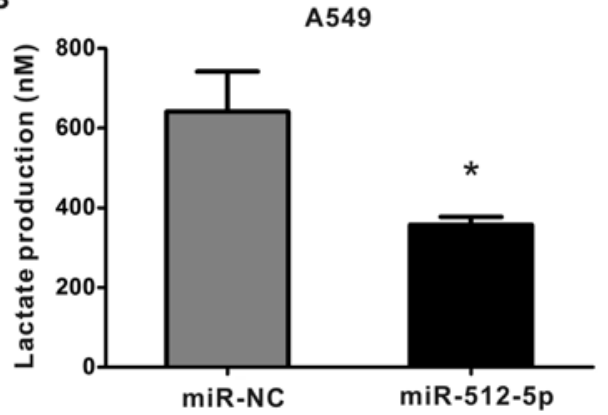

D

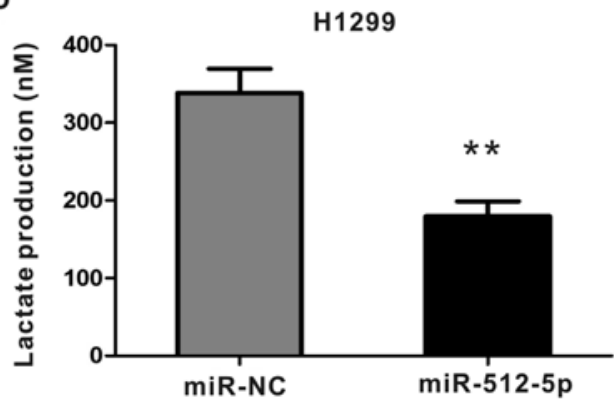

Figure 4. miR-512-5p inhibits glycolysis in NSCLC cells. The cells were transfected with miR-NC (control) or miR-512-5p mimics for $4 \mathrm{~h}$, then the culture medium was changed to RPMI-1640 without FBS. Glucose consumption (A and C) and lactate production (B and D) were detected $36 \mathrm{~h}$ later. The histograms represent mean $\pm \mathrm{SD}$ of at least three separate experiments. ${ }^{*} \mathrm{P}<0.05$ and ${ }^{* *} \mathrm{P}<0.01$.

A

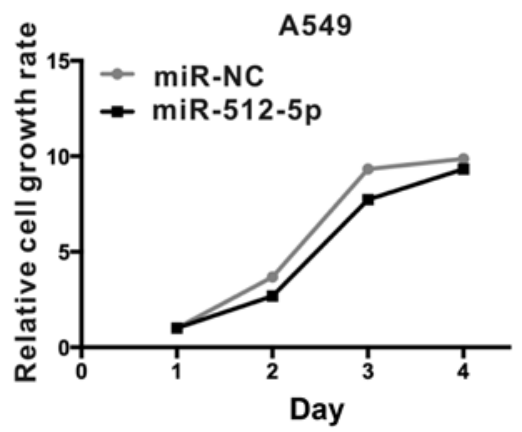

B

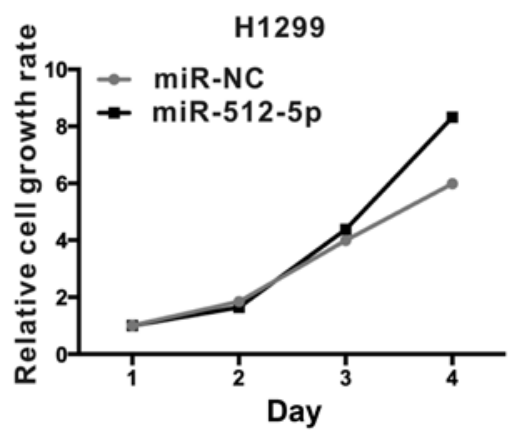

C

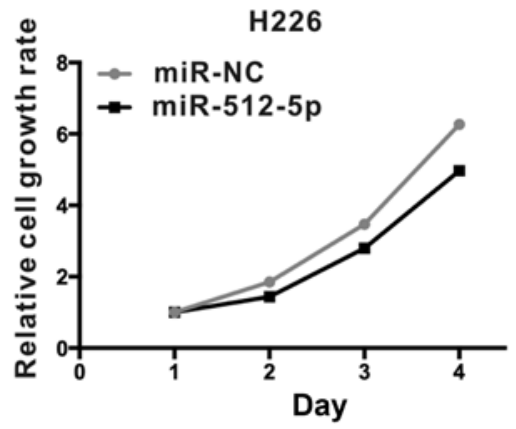

Figure 5. miR-512-5p shows no effect on cell proliferation in NSCLC cells. The cells were transfected with miR-NC (control) or miR-512-5p mimics, and cells were seeded into a 96 -well plate after $24 \mathrm{~h}$ of transfection. Cell growth rate was assessed at day 1, 2, 3 and 4 by CCK-8 assay. (A) Compared with miR-NC, miR-512-5p mimics had no influence on A549 cells. (B) miR-512-5p had no effect at day 1,2 and 3, and slightly promoted cell growth at day 4 on H1299. (C) miR-512-5p slightly inhibited cell growth on H226 compared with miR-NC, but there was no statistical differences between the treatments.

a computational screen for genes with complementary sites to $\mathrm{miR}-512-5 \mathrm{p}$ in its 3'-UTR using open-access software including TargetScan (www.targetscan.org), PicTar (http:// pictar.bio.nyu.edu), Sanger microRNA target (http://microrna. sanger) to check if p21 was a target of miR-512-5p. However, no binding site in p21 was identified. We then carried out a blast to examine whether there was complementary site between $\mathrm{p} 21$ 3'-UTR and miR-512-5p. We found a potential binding site of miR-512-5p within p21 3'-UTR (Fig. 7C). To test whether p21 is a direct target of miR-512-5p, mutation in the potential binding site was generated in the p21 3'-UTR sequence of in the complementary site (Fig. 7C). p21 3'-UTR fragment containing wild-type or mutant type binding site was cloned into pGL3prometor vector. Luciferase reporter construct containing wild-type 3'-UTR was cotransfected with pRL-TK, as well as miR-NC or miR-512-5p mimics. Furthermore, luciferase reporter construct containing wild-type 3'-UTR or mutant type 3'-UTR was cotransfected with miR-512-5p mimics and pRL-TK. As shown in Fig. 7D, the luciferase activity of wild-type 3'-UTR cotransfected with miR-512-5p decreased by $36 \%$ compared with the wild-type 3'-UTR cotransfected with miR-NC. Whereas, the luciferase activity of miR-512-5p cotransfected with wild-type p21 3'-UTR was decreased by $17 \%$ compared with that cotransfected with mutant-type p21 3'-UTR (Fig. 7E). Collectively, these results indicated that miR512-5p could bind to p21 3'-UTR to repress p21 expression.

Knockdown of p21 promotes apoptosis in NSCLC cells. We identified that p21 is a target gene of miR-512-5p. To evaluate how $\mathrm{p} 21$ is involved in the functioning of miR-512-5p, we used 
A
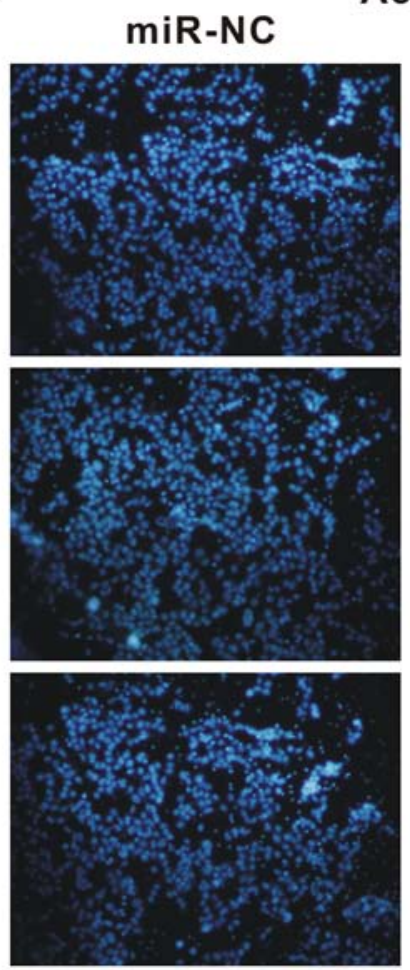

A549 miR-512-5p
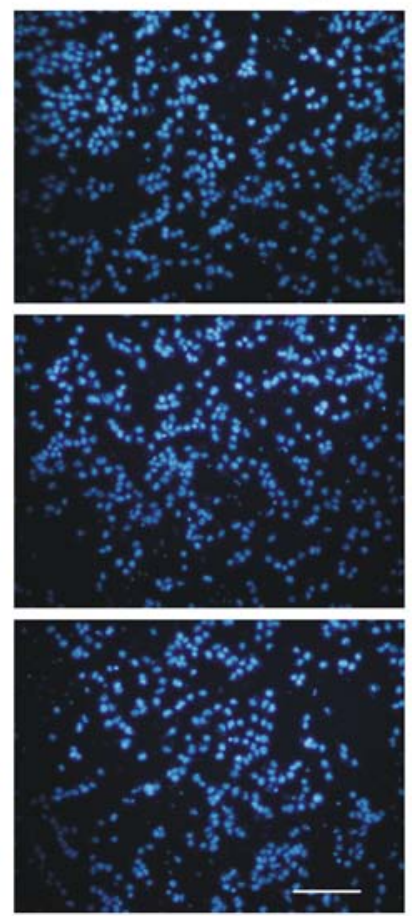

B

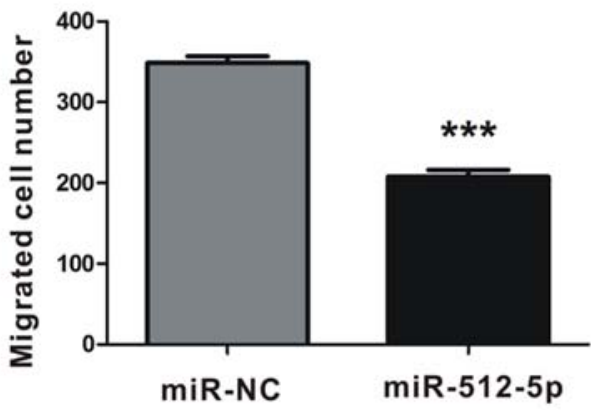

Figure 6. miR-512-5p attenuated the ability of cell migration of A549 cells. Cells (1x10 $)$ in RPMI-1640 medium were seeded into the Transwell chambers, after incubation for $72 \mathrm{~h}$, medium in chamber was removed and cells were washed, fixed with methanol, stained with DAPI, and examined. (A) Representative images of A549 cell migration under a fluorescence microscope; (B) bar graph depicting the migration; scale bar, $60 \mu \mathrm{m}$. Error bars denotes mean \pm SD, **** $\mathrm{P}<0.001$

p21 small interfering RNA (siRNA) to knock down the expression of p21 and investigated NSCLC cell apoptosis. siRNAs against p21 (sip21) were synthesized according to previous studies $(22,23)$, and the silencing efficiency was examined. A siRNA lacking homology to the genome was used as negative control. Both sip21-1 and sip21-2 had good silencing efficiency in A549 and H1299 cells, and sip21-2 showed better silencing efficiency (Fig. 8A and B). Thus, we used sip21-2 (sip21 for short) for further analyses. Cell apoptosis assay was then performed. As shown in Fig. 8C and D, knockdown of p21 significantly promoted cell apoptosis in A549 and H1299 cells. The results were in congruent with miR-512-5p overexpression.

Knockdown of miR-512-5p in H1299 cells stably expressing miR-512 attenuates apoptosis and the effect could be reversed by 21 siRNA. To confirm that miR-512-5p may affect cell apoptosis through targeting p21, we also detected the impact of p21 siRNA on cell apoptosis in the presence of miR-512-5p inhibitor in H1299 cells stably expressing miR-512-5p. To clone miR-512-5p into pEGP-miR vector, we amplified a 300 bp genomic fragment containing miR-512-5p according to the instruction. This fragment contains the precursor of miR512-3p, which can also transcribe miR-512-3p. We selected H1299 cells stably expressing miR-512 (designated as H512) or EGP control. To avoid clone-specific effects, we used bulkselected cells for functional analysis.

We then detected the impact of p21 siRNA on cell apoptosis in the presence of miR-512-5p inhibitor in H512. The efficiency of miR-512-5p inhibitor in knocking down miR-512-5p expression was examined by RT-PCR. The result showed miR-512-5p inhibitor inhibited $82 \%$ of miR-512-5p expression compared with inhibitor NC (data not shown). miR-512-5p inhibitor was transfected into H512 to knockdown the expression of miR-512-5p, and apoptosis assays were performed. As shown in Fig. 9, miR-512-5p inhibitor attenuated apoptosis in H512 cells (Fig. 9A and B). In the presence of p21 siRNA, the effect of miR-512-5p inhibitor on apoptosis could be rescued (Fig. 9C and D).

Knockdown of p21 decreases glycolysis in NSCLC cells. To further verify that miR-512-5p affected NSCLC cell glycolysis through targeting p21, we transfected A549 and H1299 with sip21 for $4 \mathrm{~h}$ and then the culture medium was changed to RPMI-1640 without FBS. After incubation for $36 \mathrm{~h}$, we detected glucose consumption and lactate production. As expected, the rates of glucose consumption and lactate production were strongly decreased by sip21 in A549 cells (Fig. 10A and B). In H1299 cells, glucose consumption showed a trend of decreasing (Fig. 10C), while the lactate production had a significant decrease (Fig. 10D). The results were consistent with $\mathrm{miR}-512-5 \mathrm{p}$ overexpression.

\section{Discussion}

miR-512-5p is the $5^{\prime}$ terminal of miR-512 precursor. It was reported that epigenetic treatment could rescue the expression of miR-512-5p, and induce gastric cancer cell apoptosis (14). Reactivation of both miR-512-5p and miR-373 was able 
A

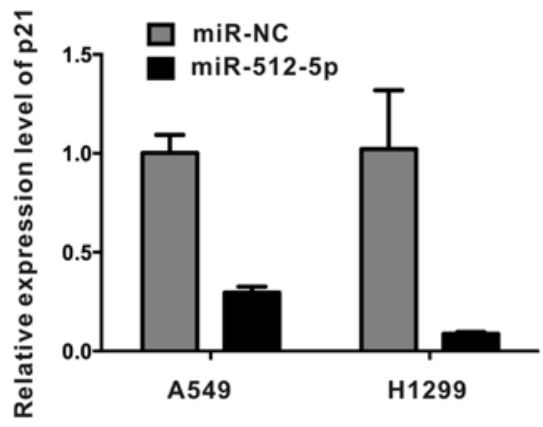

B

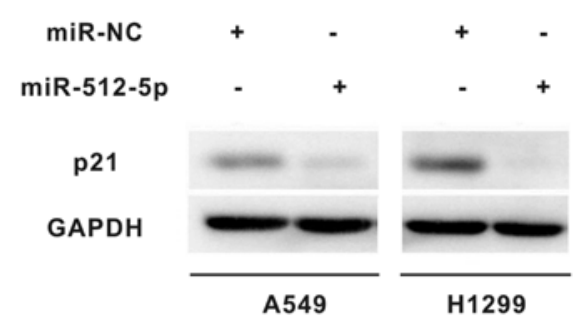

C

\begin{tabular}{|c|c|}
\hline hsa-miR-512-5p & 3'- CUUUCACGGGAGUUCCGACUCAC -5 \\
\hline p21 3'-UTR & AGCU \\
\hline GL3-p & \\
\hline
\end{tabular}

D

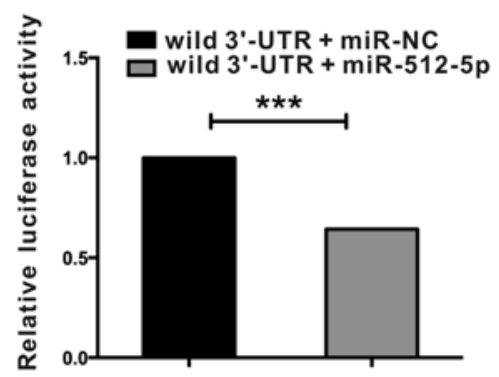

E

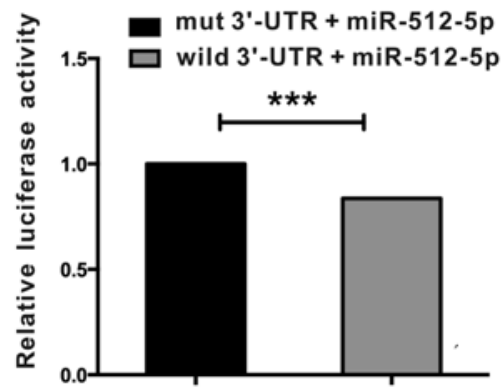

Figure 7. miR-512-5p targets p21 directly. (A) Expression level of p21 mRNA and protein (B) were detected by real-time PCR and western blotting in A549 and H1299 cells. GAPDH was taken as internal control. The experiments showed miR-512-5p inhibited the expression of p21 in NSCLC cells. (C) Sequence of the miR-512-5p binding site within p21 3'-UTR was predicted, and mutation was generated in the p21 3'-UTR sequence of the complementary site. (D) The p21 3'-UTR fragment containing wild-type binding site was cloned into pGL3-promoter vector. The luciferase reporter construct containing wild-type 3'-UTR was cotransfected with pRL-TK, as well as miR-NC or miR-512-5p mimics. The luciferase activity of wild-type 3'-UTR cotransfected with miR-512-5p decreased significantly compared with that cotransfected with miR-NC. (E) p21 3'-UTR fragment containing wild-type or mutant-type binding site was cloned into pGL3-promoter vector. Luciferase reporter construct containing wild-type 3'-UTR or mutant type 3'-UTR was cotransfected with miR-512-5p mimics and pRL-TK. The luciferase activity of wild-type p21 3'-UTR cotransfected with miR-512-5p decreased significantly compared with that cotransfected with mutant type 3 '-UTR. Error bars represent mean $\pm \mathrm{SD},{ }^{* * *} \mathrm{P}<0.001$.

to accelerate cisplatin-induced apoptosis inhibiting cell migration (24).

In this study, we first detected the expression of miR-512-5p in 12 paired normal lung tissues and NSCLC tumor tissues. We found that the expression of miR-512-5p was downregulated in $83 \%$ (10 out of 12) of the NSCLC patient samples, which suggested miR-512-5p acts as a tumor suppressor. Retinoic acid (RA) played an important role in cell differentiation, cell proliferation and cell apoptosis (25). Previous studies showed dysregulation of RA pathway was related to tumorigenesis, including lung cancer (26). Thus, we treated NSCLC cells with RA to test whether RA affect the expression of miR-512-5p. Real-time PCR showed that when A549 and H1299 cells were exposed to $10 \mu \mathrm{M}$ RA or $100 \mu \mathrm{M}$ RA, the expression of miR-512-5p increased.

To investigate the influence of miR-512-5p on NSCLC cells, we overexpressed miR-512-5p by transient transfection. We found that overexpression of miR-512-5p promoted cell apoptosis, inhibited cell glycolysis and attenuated A549 cells migration, but had no effect on cell proliferation in A549, H1299 and H226 cells. Adi Harel and colleagues (24) reported miR-512-5p inhibited A549 cells proliferation, which was not consistent with our study. A plausible but yet-to-be validated possibility is that the different cell culture condition may attribute to the cell proliferation in A549 cells in the presence of miR-512-5p.

To overcome bioenergetic and biosynthetic demands of abnormal proliferation, cancer cells must reprogram their metabolism and become more dependent on aerobic glycolysis (27). Recent studies reported that low intracellular concentration of glucose may induce ATP depletion, stimulate mitochondrial death pathway cascade, and may induce oxidative stress and trigger of bax-associated events (28-30). Taken together with the function of miR-512-5p in cell apoptosis and cell glycolysis, the inhibition of glucose uptake by miR-512-5p might play a role in the development of NSCLC cells.

To illuminate the effect of miR-512-5p in NSCLC cells, we identified that p21 is a new target of miR-512-5p. Although open-access software did not reveal p21 as a miR-512-5p target, blast screen did find a miR-512-5p complementary site in the p21 3'-UTR. The complementary site mainly includes the seed region of miR-512-5p, as well as a few bases in the 3 ' region. The luciferase reporter assay confirmed that the disruption of complementary site in miR-512-5p seed region 
A

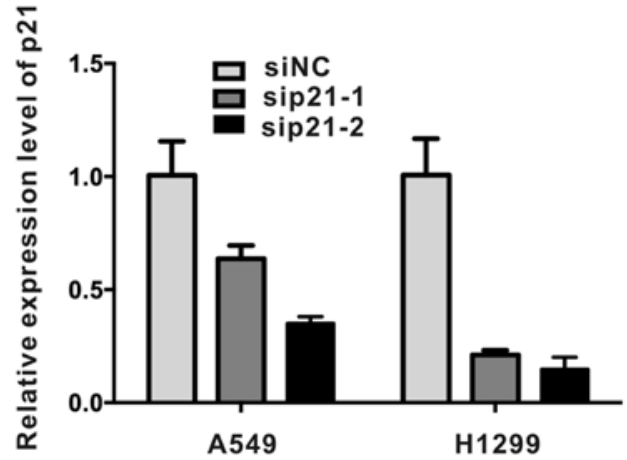

C

A549
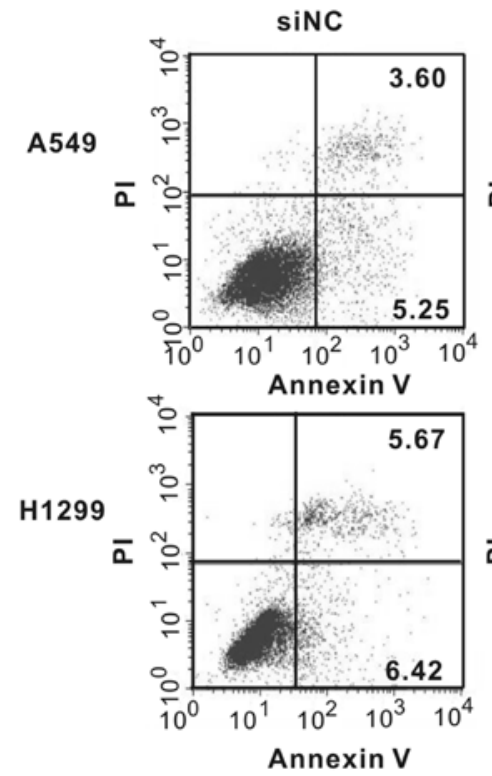
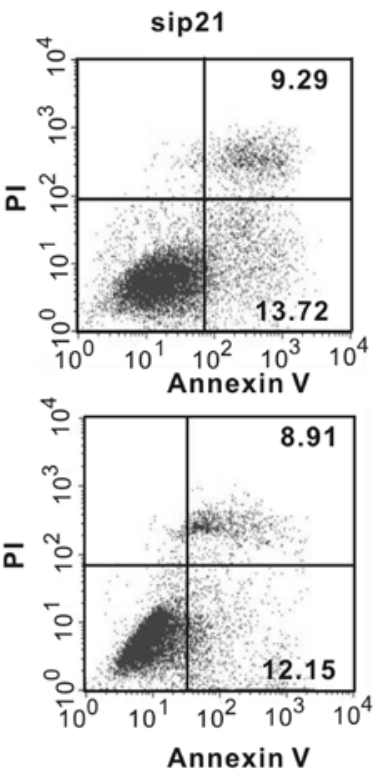

B

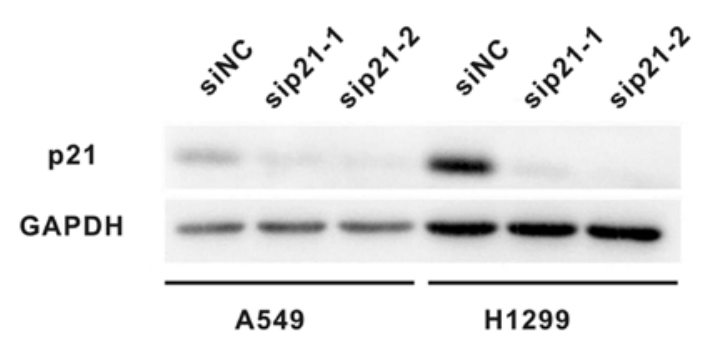

D

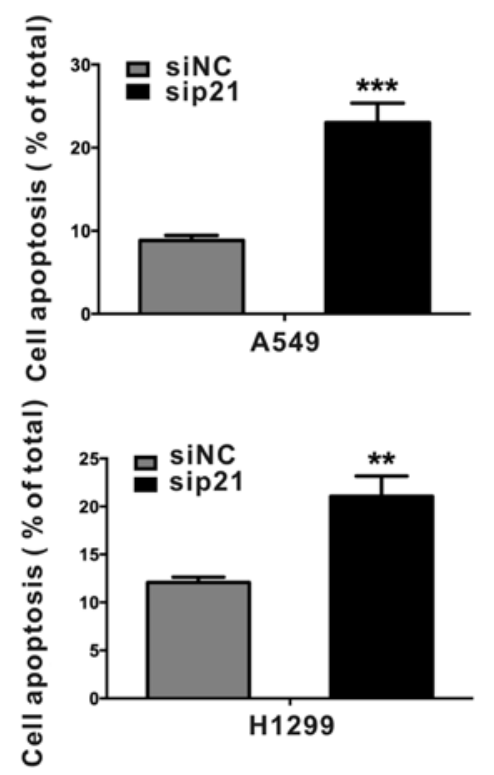

Figure 8. Knockdown of p21 promotes NSCLC cells apoptosis. (A) siRNAs targeting p21 were transfected into A549 and H1299 cells. RNA was isolated 48 h after transfection, and the silencing efficiency was detected by RT-PCR. (B) Western blotting was used to detect the silencing efficiency of sip21-1 and sip21-2 on p21 protein level in A549 and H1299 cells. p21 siRNA-2 (sip21-2) showed better efficiency compared to sip21-1, thus we use sip21-2 (sip21 for short) for further study. Apoptosis assays were performed $48 \mathrm{~h}$ after transfection of A549 cells or H1299 cells with $50 \mathrm{nM}$ with siRNA control (siNC) or siRNA against p21 (sip21). (C) Representative percentage of apoptotic cells using flow cytometric analysis. (D) The histogram shows the apoptotic cell percentage detected by FCM, and error bars represent mean $\pm \mathrm{SD} .{ }^{* *} \mathrm{P}<0.01$ and ${ }^{* * *} \mathrm{P}<0.001$

showed higher luciferase activity compared with the wild-type binding site. Computational prediction of miRNA target genes is difficult in animal genomes due to the imperfect pairing of the miRNA with the corresponding target site and the software often generate many false positive interactions. Our result showed that Blast-based method might help predicting the miRNA target.

As a member of the cyclin-dependent kinase inhibitors, p21 plays a significant role in cell cycle by acting as cyclin kinase inhibitor (CKI). p21 also directly blocks DNA replication and mediates DNA repair processes by binding to proliferating cell nuclear antigen (PCNA) (31). In addition, p21 associates directly with E2F1 (32), STAT3 (33) and MYC (34) thereby suppressing transcription activities.

Although best known for its important role in growthinhibition, p21 also protects cells against apoptosis in growth factor deprivation, p53 overexpression and during the differentiation of monocytes (35). Under these conditions, cytoplasmically localized p21 may bind to and attenuate the activity of proteins directly associated with apoptosis, including apoptosis signal- regulating kinase 1 (ASK1), stress-activated protein kinases (SAPKs), procaspase 3 , caspase 8 and caspase $10(35,36)$. However, p21 might improve apoptosis under certain stimuli, such as activation of tumor necrosis factor family of death receptors or effects on DNA repair (37). Therefore, the function of p21 depends on the specific circumstances, suggests that it can be a tumor suppressor or an oncogene.

We found knockdown of p21 promoted cell apoptosis, and decreased cell glycolysis. The results were consistent with miR-512-5p overexpression. However, p21siRNA exhibited a more obvious effect on cell apoptosis in A549 and H1299 cells. It is well known that a single miRNA can bind to and regulate many different mRNA targets. miR-512-5p may target other genes to regulate cell apoptosis, which might neutralize the effect of targeting p21. We also tested the effect of miR-512-5p in H1299 cells stably expressing miR-512. miR-512-5p inhibitor significantly depressed cell apoptosis, and in the presence of miR-512-5p inhibitor, p21siRNA was able to rescue cell apoptosis. The results revealed miR-512-5p induces NSCLC cell apoptosis by directly regulating p21. 
A

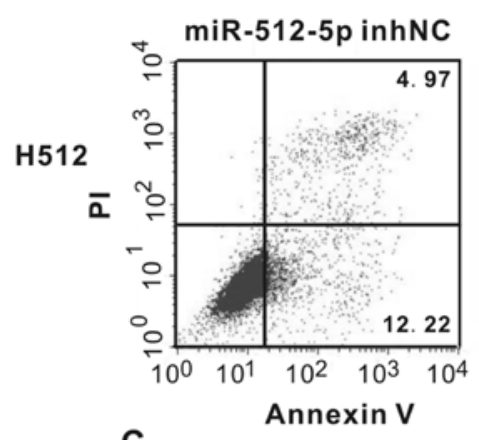

C

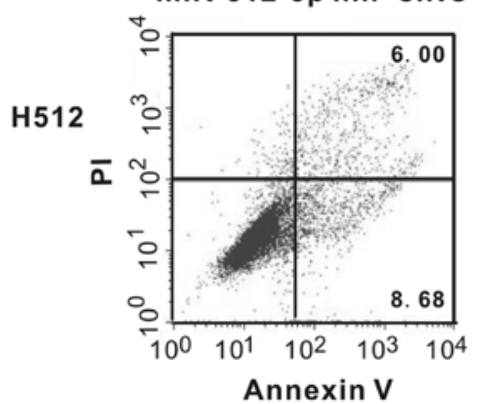

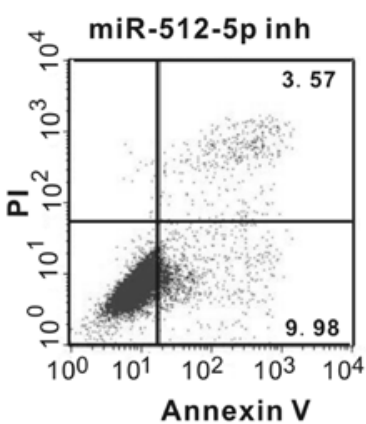

miR-512-5p inh+sip21

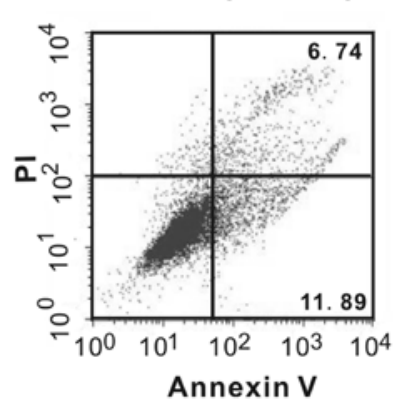

B

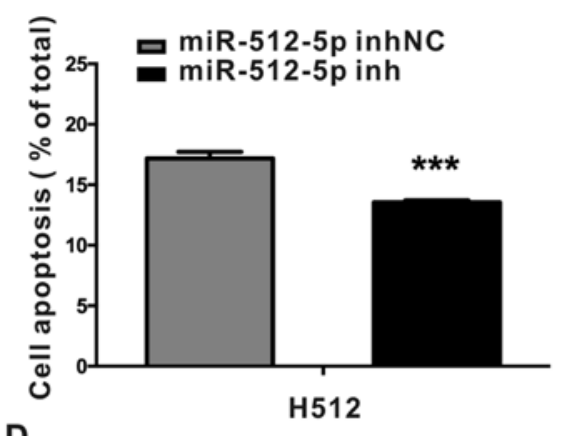

。

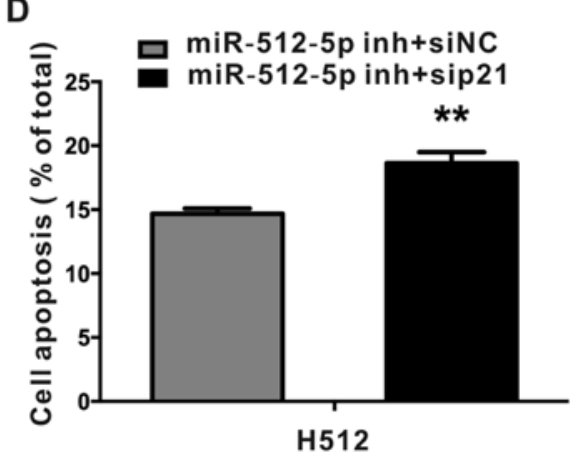

Figure 9. Inhibitor of miR-512-5p attenuates apoptosis in H1299 cells stably expressing miR-512, the effect could be reversed by siRNA against p21. (A) Representative percentage of apoptotic cells using flow cytometric analysis. Apoptosis assays were performed $48 \mathrm{~h}$ after transfection of $\mathrm{H} 512 \mathrm{with}$ miR-512-5p inhibitor (miR-512-5p inh) or inhibitor NC. (B) The histogram of (A) showed the apoptotic cell percentage detected by FCM. In H512 cells, miR-512-5p inhibitor significantly depressed cell apoptosis. (C) Representative percentage of apoptotic cells using flow cytometric analysis. Apoptosis assays were performed $48 \mathrm{~h}$ after transfection of $\mathrm{H} 512$ with miR-512-5p inh, as well as siNC or sip21. (D) The histogram of (C) shows the apoptotic cell percentage detected by FCM. In the presence of sip21, the effect of miR-512-5p inhibitor on apoptosis could be rescued. Each assay was repeated three times. Error bars represent mean $\pm \mathrm{SD}$. ${ }^{* *} \mathrm{P}<0.01$ and ${ }^{* * * *} \mathrm{P}<0.001$.

A

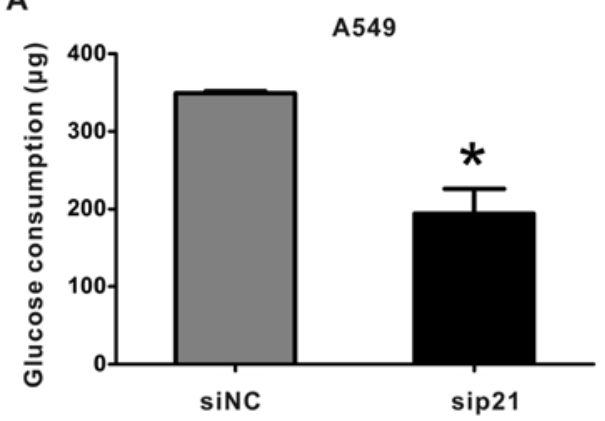

C

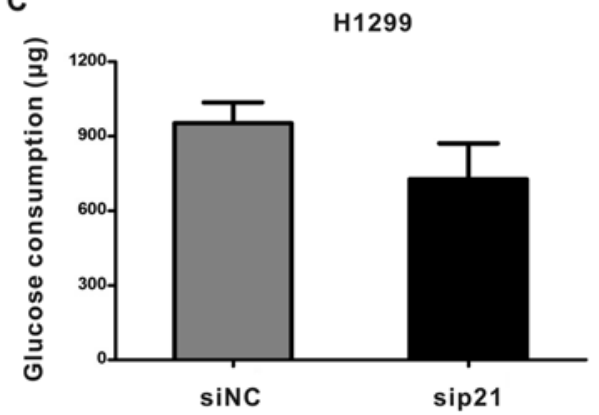

B

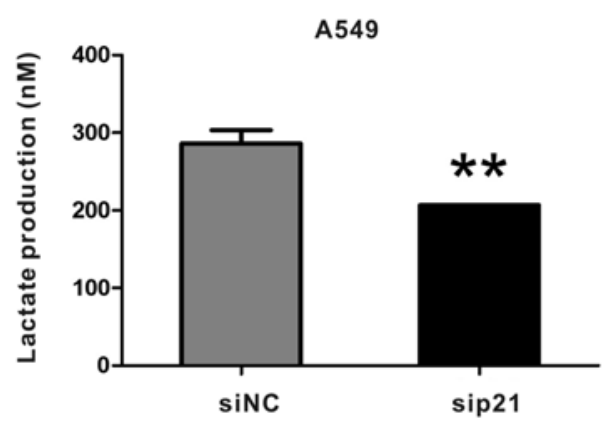

D

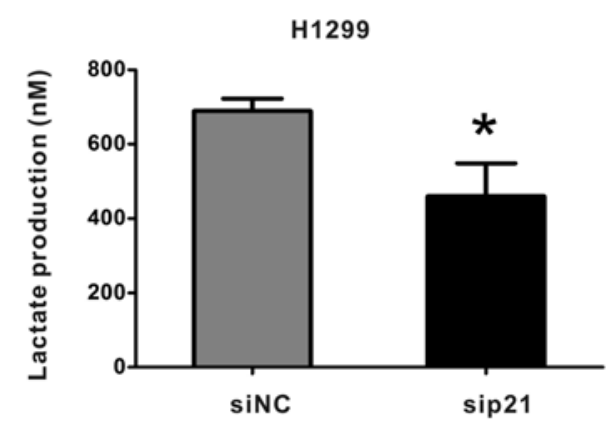

Figure 10. Knockdown of p21 decreased glycolysis in NSCLC cells. The cells were transfected with siRNA control (siNC) or siRNA against p21 (sip21) for $4 \mathrm{~h}$, then the culture medium was changed to RPMI-1640 without FBS. Glucose consumption (A and C) and lactate production (B and D) were detected $36 \mathrm{~h}$ later. The histograms represent mean $\pm \mathrm{SD}$ of at least three separate experiments. ${ }^{*} \mathrm{P}<0.05$ and ${ }^{* *} \mathrm{P}<0.01$.

In conclusion, our study demonstrates miR-512-5p induced NSCLC cells apoptosis and inhibited glycolysis by regulating p21 in A549 and H1299 cells. We also found that miR-512-5p is decreased in $83 \%(10 / 12)$ of NSCLC patient lung cancer 
samples compared with normal sample from the same patient, which suggests that miR-512-5p may act as a tumor suppressor in NSCLC.

\section{Acknowledgements}

This study was supported by Shanghai Municipal Natural Science foundation 14ZR1403700.

\section{References}

1. Torre LA, Bray F, Siegel RL, Ferlay J, Lortet-Tieulent J and Jemal A: Global cancer statistics, 2012. CA Cancer J Clin 65: 87-108, 2015

2. Li J, Bi L, Sun Y, Lu Z, Lin Y, Bai O and Shao H: Text mining and network analysis of molecular interaction in non-small cel lung cancer by using natural language processing. Mol Biol Rep 41: 8071-8079, 2014.

3. Joshi P, Jeon YJ, Laganà A, Middleton J, Secchiero P, Garofalo M and Croce CM: MicroRNA-148a reduces tumorigenesis and increases TRAIL-induced apoptosis in NSCLC. Proc Natl Acad Sci USA 112: 8650-8655, 2015.

4. Li Y,Zhang D, Chen C, Ruan Z,Li Y and Huang Y: MicroRNA-212 displays tumor-promoting properties in non-small cell lung cancer cells and targets the hedgehog pathway receptor PTCH1. Mol Biol Cell 23: 1423-1434, 2012.

5. Mendell JT: miRiad roles for the miR-17-92 cluster in development and disease. Cell 133: 217-222, 2008.

6. Johnson SM, Grosshans H, Shingara J, Byrom M, Jarvis R, Cheng A, Labourier E, Reinert KL, Brown D and Slack FJ: RAS is regulated by the let-7 microRNA family. Cell 120: 635-647, 2005 .

7. Loo JM, Scherl A, Nguyen A, Man FY, Weinberg E, Zeng Z, Saltz L, Paty PB and Tavazoie SF: Extracellular metabolic energetics can promote cancer progression. Cell 160: 393-406, 2015.

8. Okazawa H, Shimizu J, Kamei M, Imafuku I, Hamada H and Kanazawa I: Bcl-2 inhibits retinoic acid-induced apoptosis during the neural differentiation of embryonal stem cells. J Cell Biol 132: 955-968, 1996.

9. Burnett A, Wetzler M and Löwenberg B: Therapeutic advances in acute myeloid leukemia. J Clin Oncol 29: 487-494, 2011.

10. Fisher JN, Terao M, Fratelli M, Kurosaki M, Paroni G, Zanetti A, Gianni M, Bolis M, Lupi M, Tsykin A, et al: MicroRNA networks regulated by all-trans retinoic acid and Lapatinib control the growth, survival and motility of breast cancer cells. Oncotarget 6: 13176-13200, 2015.

11. Khan S, Wall D, Curran C, Newell J, Kerin MJ and Dwyer RM: MicroRNA-10a is reduced in breast cancer and regulated in part through retinoic acid. BMC Cancer 15: 345, 2015.

12. Lichner Z, Páll E, Kerekes A, Pállinger E, Maraghechi P, Bosze Z and Gócza E: The miR-290-295 cluster promotes pluripotency maintenance by regulating cell cycle phase distribution in mouse embryonic stem cells. Differentiation 81: 11-24, 2011.

13. Cheung TH, Man KN, Yu MY, Yim SF, Siu NS, Lo KW, Doran G, Wong RR, Wang VW, Smith DI, et al: Dysregulated microRNAs in the pathogenesis and progression of cervical neoplasm. Cell Cycle 11: 2876-2884, 2012.

14. Saito Y, Suzuki H, Tsugawa H, Nakagawa I, Matsuzaki J, Kanai Y and Hibi T: Chromatin remodeling at Alu repeats by epigenetic treatment activates silenced microRNA-512-5p with downregulation of Mcl-1 in human gastric cancer cells. Oncogene 28: 2738-2744, 2009.

15. Sherr CJ and Roberts JM: CDK inhibitors: Positive and negative regulators of G1-phase progression. Genes Dev 13: 1501-1512, 1999.

16. Deng C, Zhang P, Harper JW, Elledge SJ and Leder P: Mice lacking p21CIP1/WAF1 undergo normal development, but are defective in G1 checkpoint control. Cell 82: 675-684, 1995.
17. Harper JW, Adami GR, Wei N, Keyomarsi K and Elledge SJ: The p21 Cdk-interacting protein Cip1 is a potent inhibitor of G1 cyclin-dependent kinases. Cell 75: 805-816, 1993.

18. Abbas T and Dutta A: p21 in cancer: Intricate networks and multiple activities. Nat Rev Cancer 9: 400-414, 2009.

19. Zhu X, Gao G, Chu K, Yang X, Ren S, Li Y, Wu H, Huang Y and Zhou C: Inhibition of RAC1-GEF DOCK3 by miR-512-3p contributes to suppression of metastasis in non-small cell lung cancer. Int J Biochem Cell Biol 61: 103-114, 2015.

20. Vander Heiden MG, Cantley LC and Thompson CB: Understanding the Warburg effect: The metabolic requirements of cell proliferation. Science 324: 1029-1033, 2009.

21. Chen J and Thompson LU: Lignans and tamoxifen, alone or in combination, reduce human breast cancer cell adhesion, invasion and migration in vitro. Breast Cancer Res Treat 80: 163-170, 2003.

22. Zhang J, Attar E, Cohen K, Crumpacker C and Scadden D: Silencing p21(Waf1/Cip1/Sdi1) expression increases gene transduction efficiency in primitive human hematopoietic cells. Gene Ther 12: 1444-1452, 2005.

23. Rao A, Coan A, Welsh JE, Barclay WW, Koumenis C and Cramer SD: Vitamin D receptor and p21/WAF1 are targets of genistein and 1,25-dihydroxyvitamin D3 in human prostate cancer cells. Cancer Res 64: 2143-2147, 2004.

24. Adi Harel S, Bossel Ben-Moshe N, Aylon Y, Bublik DR, Moskovits N, Toperoff G, Azaiza D, Biagoni F, Fuchs G, Wilder S, et al: Reactivation of epigenetically silenced miR-512 and miR-373 sensitizes lung cancer cells to cisplatin and restricts tumor growth. Cell Death Differ 22: 1328-1340, 2015.

25. di Masi A, Leboffe L, De Marinis E, Pagano F, Cicconi L, Rochette-Egly C, Lo-Coco F, Ascenzi P and Nervi C: Retinoic acid receptors: From molecular mechanisms to cancer therapy. Mol Aspects Med 41: 1-115, 2015.

26. Chen F, Cao Y, Qian J, Shao F, Niederreither K and Cardoso WV: A retinoic acid-dependent network in the foregut controls formation of the mouse lung primordium. J Clin Invest 120: 2040-2048, 2010.

27. Jones RG and Thompson CB: Tumor suppressors and cell metabolism: A recipe for cancer growth. Genes Dev 23: 537-548, 2009.

28. Muñoz-Pinedo C, El Mjiyad N and Ricci JE: Cancer metabolism: Current perspectives and future directions. Cell Death Dis 3: e248, 2012.

29. El Mjiyad N, Caro-Maldonado A, Ramírez-Peinado S and Muñoz-Pinedo C: Sugar-free approaches to cancer cell killing. Oncogene 30: 253-264, 2011.

30. Raina K, Agarwal C, Wadhwa R, Serkova NJ and Agarwal R: Energy deprivation by silibinin in colorectal cancer cells: A double-edged sword targeting both apoptotic and autophagic machineries. Autophagy 9: 697-713, 2013.

31. Li R, Waga S, Hannon GJ, Beach D and Stillman B: Differential effects by the p21 CDK inhibitor on PCNA-dependent DNA replication and repair. Nature 371: 534-537, 1994.

32. Delavaine L and La Thangue NB: Control of E2F activity by p21Waf1/Cip1. Oncogene 18: 5381-5392, 1999.

33. Coqueret O and Gascan H: Functional interaction of STAT3 transcription factor with the cell cycle inhibitor p21WAF1/CIP1/ SDI1. J Biol Chem 275: 18794-18800, 2000.

34. Kitaura H, Shinshi M, Uchikoshi Y, Ono T, Iguchi-Ariga SM and Ariga $\mathrm{H}$ : Reciprocal regulation via protein-protein interaction between c-Myc and p21(cip1/waf1/sdi1) in DNA replication and transcription. J Biol Chem 275: 10477-10483, 2000.

35. Roninson IB: Oncogenic functions of tumour suppressor p21(Waf1/Cip1/Sdi1): Association with cell senescence and tumour-promoting activities of stromal fibroblasts. Cancer Lett 179: 1-14, 2002.

36. Dotto GP: p21(WAF1/Cip1): More than a break to the cell cycle? Biochim Biophys Acta 1471: M43-M56, 2000.

37. Gartel AL: The conflicting roles of the cdk inhibitor $\mathrm{p} 21$ (CIP1/ WAF1) in apoptosis. Leuk Res 29: 1237-1238, 2005. 